\title{
Longitudinal Double-Spin Asymmetry and Cross Section for Inclusive Jet Production in Polarized Proton Collisions at $\sqrt{s}=200 \mathrm{GeV}$
}

B. I. Abelev, ${ }^{50}$ M. M. Aggarwal, ${ }^{30}$ Z. Ahammed, ${ }^{45}$ J. Amonett, ${ }^{20}$ B. D. Anderson, ${ }^{20}$ M. Anderson, ${ }^{6}$ D. Arkhipkin, ${ }^{13}$ G. S. Averichev, ${ }^{12}$ Y. Bai, ${ }^{28}$ J. Balewski, ${ }^{17}$ O. Barannikova, ${ }^{9}$ L. S. Barnby, ${ }^{2}$ J. Baudot, ${ }^{18}$ S. Bekele, ${ }^{29}$ V. V. Belaga, ${ }^{12}$ A. Bellingeri-Laurikainen, ${ }^{40}$ R. Bellwied ${ }^{48}$ F. Benedosso, ${ }^{28}$ S. Bhardwaj,${ }^{35}$ A. Bhasin, ${ }^{19}$ A. K. Bhati, ${ }^{30} \mathrm{H}$. Bichsel, ${ }^{47}$ J. Bielcik, ${ }^{50}$ J. Bielcikova, ${ }^{50}$ L. C. Bland, ${ }^{3}$ S-L. Blyth, ${ }^{22}$ B. E. Bonner,${ }^{36}$ M. Botje, ${ }^{28}$ J. Bouchet,${ }^{40}$ A. V. Brandin, ${ }^{26}$ A. Bravar, ${ }^{3}$ T. P. Burton, ${ }^{2}$ M. Bystersky, ${ }^{11}$ R. V. Cadman, ${ }^{1}$ X. Z. Cai,${ }^{39}$ H. Caines,${ }^{50}$ M. Calderón de la Barca Sánchez, ${ }^{6}$ J. Castillo, ${ }^{28}$ O. Catu ${ }^{50}$ D. Cebra, ${ }^{6}$ Z. Chajecki, ${ }^{29}$ P. Chaloupka, ${ }^{11}$ S. Chattopadhyay, ${ }^{45}$ H. F. Chen ${ }^{38}$ J. H. Chen, ${ }^{39}$ J. Cheng, ${ }^{43}$ M. Cherney ${ }^{10}$ A. Chikanian, ${ }^{50}$ W. Christie, ${ }^{3}$ J. P. Coffin, ${ }^{18}$ T. M. Cormier, ${ }^{48}$ M. R. Cosentino, ${ }^{37}$ J. G. Cramer,${ }^{47}$ H. J. Crawford, ${ }^{5}$ D. Das, ${ }^{45}$ S. Das, ${ }^{45}$ S. Dash, ${ }^{15}$ M. Daugherity, ${ }^{42}$ M. M. de Moura, ${ }^{37}$ T. G. Dedovich,${ }^{12}$ M. DePhillips, ${ }^{3}$ A. A. Derevschikov, ${ }^{32}$ L. Didenko, ${ }^{3}$ T. Dietel, ${ }^{14}$ P. Djawotho, ${ }^{17}$ S. M. Dogra, ${ }^{19}$ W. J. Dong, ${ }^{7}$ X. Dong,,${ }^{38}$ J. E. Draper, ${ }^{6}$

F. Du, ${ }^{50}$ V. B. Dunin, ${ }^{12}$ J. C. Dunlop, ${ }^{3}$ M. R. Dutta Mazumdar, ${ }^{45}$ V. Eckardt, ${ }^{24}$ W. R. Edwards, ${ }^{22}$ L. G. Efimov, ${ }^{12}$ V. Emelianov, ${ }^{26}$ J. Engelage,${ }^{5}$ G. Eppley, ${ }^{36}$ B. Erazmus,${ }^{40}$ M. Estienne, ${ }^{18}$ P. Fachini, ${ }^{3}$ R. Fatemi, ${ }^{23}$ J. Fedorisin, ${ }^{12}$ P. Filip, ${ }^{13}$ E. Finch, ${ }^{50}$ V. Fine, ${ }^{3}$ Y. Fisyak,${ }^{3}$ J. Fu,${ }^{49}$ C. A. Gagliardi, ${ }^{41}$ L. Gaillard, ${ }^{2}$ M. S. Ganti, ${ }^{45}$ V. Ghazikhanian, ${ }^{7}$ P. Ghosh,${ }^{45}$ J. E. Gonzalez, ${ }^{7}$ Y. G. Gorbunov, ${ }^{10}$ H. Gos,${ }^{46}$ O. Grebenyuk, ${ }^{28}$ D. Grosnick, ${ }^{44}$ S. M. Guertin, ${ }^{7}$ K. S. F. F. Guimaraes, ${ }^{37}$ N. Gupta, ${ }^{19}$ T. D. Gutierrez, ${ }^{6}$ B. Haag, ${ }^{6}$ T. J. Hallman, ${ }^{3}$ A. Hamed, ${ }^{48}$ J. W. Harris ${ }^{50}$ W. He,${ }^{17}$ M. Heinz,${ }^{50}$ T. W. Henry, ${ }^{41}$

S. Hepplemann, ${ }^{31}$ B. Hippolyte, ${ }^{18}$ A. Hirsch ${ }^{33}$ E. Hjort, ${ }^{22}$ A. M. Hoffman ${ }^{23}$ G. W. Hoffmann, ${ }^{42}$ M. J. Horner, ${ }^{22}$

H. Z. Huang, ${ }^{7}$ S. L. Huang, ${ }^{38}$ E. W. Hughes, ${ }^{4}$ T. J. Humanic, ${ }^{29}$ G. Igo, ${ }^{7}$ P. Jacobs,${ }^{22}$ W. W. Jacobs,${ }^{17}$ P. Jakl,${ }^{11}$ F. Jia, ${ }^{21}$ H. Jiang, ${ }^{7}$ P. G. Jones, ${ }^{2}$ E. G. Judd, ${ }^{5}$ S. Kabana ${ }^{40}$ K. Kang, ${ }^{43}$ J. Kapitan, ${ }^{11}$ M. Kaplan, ${ }^{8}$ D. Keane,${ }^{20}$ A. Kechechyan, ${ }^{12}$ V. Yu. Khodyrev, ${ }^{32}$ B. C. Kim, ${ }^{34}$ J. Kiryluk, ${ }^{23}$ A. Kisiel, ${ }^{46}$ E. M. Kislov, ${ }^{12}$ S. R. Klein, ${ }^{22}$ A. Kocoloski, ${ }^{23}$ D. D. Koetke, ${ }^{44}$

T. Kollegger, ${ }^{14}$ M. Kopytine,${ }^{20}$ L. Kotchenda, ${ }^{26}$ V. Kouchpil,${ }^{11}$ K. L. Kowalik, ${ }^{22}$ M. Kramer, ${ }^{27}$ P. Kravtsov,${ }^{26}$

V. I. Kravtsov, ${ }^{32}$ K. Krueger, ${ }^{1}$ C. Kuhn, ${ }^{18}$ A. I. Kulikov, ${ }^{12}$ A. Kumar, ${ }^{30}$ A. A. Kuznetsov, ${ }^{12}$ M. A. C. Lamont,${ }^{50}$ J. M. Landgraf, ${ }^{3}$ S. Lange, ${ }^{14}$ S. LaPointe, ${ }^{48}$ F. Laue, ${ }^{3}$ J. Lauret,${ }^{3}$ A. Lebedev, ${ }^{3}$ R. Lednicky,${ }^{13}$ C-H. Lee,${ }^{34}$ S. Lehocka, ${ }^{12}$ M. J. LeVine, ${ }^{3}$ C. Li $^{38}$ Q. Li, ${ }^{48}$ Y. Li, ${ }^{43}$ G. Lin,${ }^{50}$ X. Lin, ${ }^{49}$ S. J. Lindenbaum, ${ }^{27}$ M. A. Lisa, ${ }^{29}$ F. Liu, ${ }^{49}$ H. Liu, ${ }^{38}$ J. Liu, ${ }^{36}$ L. Liu, ${ }^{49}$ Z. Liu, ${ }^{49}$ T. Ljubicic, ${ }^{3}$ W. J. Llope,${ }^{36}$ H. Long, ${ }^{7}$ R. S. Longacre, ${ }^{3}$ W. A. Love, ${ }^{3}$ Y. Lu, ${ }^{49}$ T. Ludlam, ${ }^{3}$ D. Lynn ${ }^{3}$ G. L. Ma, ${ }^{39}$ J. G. Ma ${ }^{7}$ Y. G. Ma, ${ }^{39}$ D. Magestro, ${ }^{29}$ D.P. Mahapatra, ${ }^{15}$ R. Majka ${ }^{50}$ L. K. Mangotra, ${ }^{19}$ R. Manweiler, ${ }^{44}$

S. Margetis,${ }^{20}$ C. Markert, ${ }^{42}$ L. Martin, ${ }^{40}$ H. S. Matis, ${ }^{22}$ Yu. A. Matulenko, ${ }^{32}$ C. J. McClain, ${ }^{1}$ T. S. McShane, ${ }^{10}$

Yu. Melnick ${ }^{32}$ A. Meschanin, ${ }^{32}$ J. Millane, ${ }^{23}$ M. L. Miller, ${ }^{23}$ N. G. Minaev, ${ }^{32}$ S. Mioduszewski, ${ }^{41}$ C. Mironov, ${ }^{20}$ A. Mischke, ${ }^{28}$ D. K. Mishra,${ }^{15}$ J. Mitchell,${ }^{36}$ B. Mohanty, ${ }^{45}$ L. Molnar, ${ }^{33}$ C. F. Moore,${ }^{42}$ D. A. Morozov, ${ }^{32}$ M. G. Munhoz, ${ }^{37}$

B. K. Nandi ${ }^{16}$ C. Nattrass, ${ }^{50}$ T. K. Nayak, ${ }^{45}$ J. M. Nelson, ${ }^{2}$ P. K. Netrakanti, ${ }^{45}$ L. V. Nogach, ${ }^{32}$ S. B. Nurushev, ${ }^{32}$

G. Odyniec, ${ }^{22}$ A. Ogawa, ${ }^{3}$ V. Okorokov,${ }^{26}$ M. Oldenburg,${ }^{22}$ D. Olson, ${ }^{22}$ M. Pachr, ${ }^{11}$ S. K. Pal, ${ }^{45}$ Y. Panebratsev, ${ }^{12}$ S. Y. Panitkin, ${ }^{3}$ A. I. Pavlinov, ${ }^{48}$ T. Pawlak,${ }^{46}$ T. Peitzmann, ${ }^{28}$ V. Perevoztchikov, ${ }^{3}$ C. Perkins, ${ }^{5}$ W. Peryt, ${ }^{46}$ S. C. Phatak, ${ }^{15}$

R. Picha, ${ }^{6}$ M. Planinic, ${ }^{51}$ J. Pluta, ${ }^{46}$ N. Poljak, ${ }^{51}$ N. Porile, ${ }^{33}$ J. Porter, ${ }^{47}$ A. M. Poskanzer, ${ }^{22}$ M. Potekhin, ${ }^{3}$

E. Potrebenikova, ${ }^{12}$ B. V. K. S. Potukuchi, ${ }^{19}$ D. Prindle, ${ }^{47}$ C. Pruneau, ${ }^{48}$ J. Putschke, ${ }^{22}$ G. Rakness,${ }^{31}$ R. Raniwala,${ }^{35}$ S. Raniwala, ${ }^{35}$ R. L. Ray, ${ }^{42}$ S. V. Razin, ${ }^{12}$ J. Reinnarth,${ }^{40}$ D. Relyea, ${ }^{4}$ A. Ridiger,${ }^{26}$ H. G. Ritter, ${ }^{22}$ J. B. Roberts, ${ }^{36}$ O. V. Rogachevskiy, ${ }^{12}$ J. L. Romero, ${ }^{6}$ A. Rose,${ }^{22}$ C. Roy, ${ }^{40}$ L. Ruan, ${ }^{22}$ M. J. Russcher, ${ }^{28}$ R. Sahoo, ${ }^{15}$ T. Sakuma, ${ }^{23}$ S. Salur, ${ }^{50}$ J. Sandweiss, ${ }^{50}$ M. Sarsour, ${ }^{41}$ P. S. Sazhin, ${ }^{12}$ J. Schambach, ${ }^{42}$ R. P. Scharenberg, ${ }^{33}$ N. Schmitz ${ }^{24}$ J. Seger, ${ }^{10}$ I. Selyuzhenkov, ${ }^{48}$ P. Seyboth, ${ }^{24}$ A. Shabetai, ${ }^{20}$ E. Shahaliev, ${ }^{12}$ M. Shao, ${ }^{38}$ M. Sharma,${ }^{30}$ W. Q. Shen, ${ }^{39}$ S. S. Shimanskiy, ${ }^{12}$

E. P. Sichtermann, ${ }^{22}$ F. Simon, ${ }^{23}$ R. N. Singaraju, ${ }^{45}$ N. Smirnov,${ }^{50}$ R. Snellings, ${ }^{28}$ G. Sood, ${ }^{44}$ P. Sorensen, ${ }^{3}$ J. Sowinski, ${ }^{17}$

J. Speltz, ${ }^{18}$ H. M. Spinka, ${ }^{1}$ B. Srivastava, ${ }^{33}$ A. Stadnik, ${ }^{12}$ T. D. S. Stanislaus,,${ }^{44}$ R. Stock, ${ }^{14}$ A. Stolpovsky, ${ }^{48}$

M. Strikhanov, ${ }^{26}$ B. Stringfellow, ${ }^{33}$ A. A. P. Suaide, ${ }^{37}$ E. Sugarbaker, ${ }^{29}$ M. Sumbera, ${ }^{11}$ Z. Sun, ${ }^{21}$ B. Surrow, ${ }^{23}$

M. Swanger, ${ }^{10}$ T. J. M. Symons, ${ }^{22}$ A. Szanto de Toledo, ${ }^{37}$ A. Tai, ${ }^{7}$ J. Takahashi, ${ }^{37}$ A. H. Tang, ${ }^{3}$ T. Tarnowsky, ${ }^{33}$ D. Thein, ${ }^{7}$ J.H. Thomas, ${ }^{22}$ A. R. Timmins, ${ }^{2}$ S. Timoshenko, ${ }^{26}$ M. Tokarev, ${ }^{12}$ T. A. Trainor, ${ }^{47}$ S. Trentalange, ${ }^{7}$ R. E. Tribble, ${ }^{41}$

O. D. Tsai, ${ }^{7}$ J. Ulery, ${ }^{33}$ T. Ullrich, ${ }^{3}$ D. G. Underwood, ${ }^{1}$ G. Van Buren,${ }^{3}$ N. van der Kolk, ${ }^{28}$ M. van Leeuwen, ${ }^{22}$

A. M. Vander Molen, ${ }^{25}$ R. Varma, ${ }^{16}$ I. M. Vasilevski, ${ }^{13}$ A. N. Vasiliev, ${ }^{32}$ R. Vernet, ${ }^{18}$ S. E. Vigdor, ${ }^{17}$ Y. P. Viyogi, ${ }^{15}$

S. Vokal, ${ }^{12}$ S. A. Voloshin, ${ }^{48}$ W. T. Waggoner, ${ }^{10}$ F. Wang, ${ }^{33}$ G. Wang, ${ }^{7}$ J. S. Wang, ${ }^{21}$ X. L. Wang, ${ }^{38}$ Y. Wang, ${ }^{43}$

J. W. Watson, ${ }^{20}$ J. C. Webb, ${ }^{44}$ G. D. Westfall, ${ }^{25}$ A. Wetzler, ${ }^{22}$ C. Whitten, Jr., ${ }^{7}$ H. Wieman, ${ }^{22}$ S. W. Wissink, ${ }^{17}$ R. Witt,${ }^{50}$

J. Wood, ${ }^{7}$ J. Wu, ${ }^{38}$ N. Xu, ${ }^{22}$ Q. H. Xu, ${ }^{22}$ Z. Xu, ${ }^{3}$ P. Yepes,${ }^{36}$ I-K. Yoo, ${ }^{34}$ V. I. Yurevich, ${ }^{12}$ W. Zhan, ${ }^{21}$ H. Zhang, ${ }^{3}$ 
W. M. Zhang, ${ }^{20}$ Y. Zhang, ${ }^{38}$ Z. P. Zhang, ${ }^{38}$ Y. Zhao,${ }^{38}$ C. Zhong, ${ }^{39}$ R. Zoulkarneev, ${ }^{13}$ Y. Zoulkarneeva, ${ }^{13}$ A. N. Zubarev, ${ }^{12}$ and J. X. Zuo ${ }^{39}$

(STAR Collaboration)

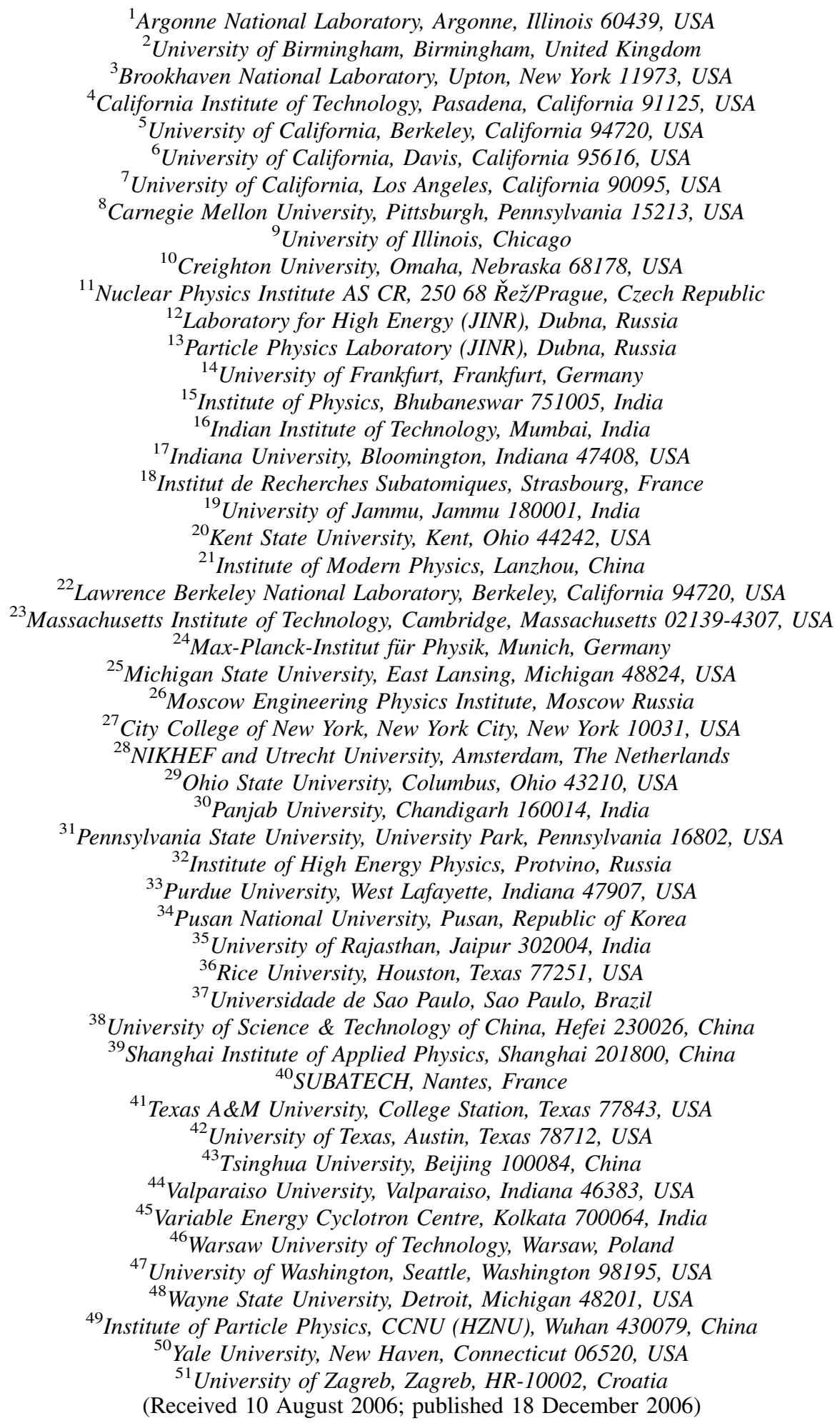

We report a measurement of the longitudinal double-spin asymmetry $A_{\mathrm{LL}}$ and the differential cross section for inclusive midrapidity jet production in polarized proton collisions at $\sqrt{s}=200 \mathrm{GeV}$. The cross 
section data cover transverse momenta $5<p_{T}<50 \mathrm{GeV} / c$ and agree with next-to-leading order perturbative QCD evaluations. The $A_{\mathrm{LL}}$ data cover $5<p_{T}<17 \mathrm{GeV} / c$ and disfavor at $98 \%$ C.L. maximal positive gluon polarization in the polarized nucleon.

Deep-inelastic scattering (DIS) experiments with polarized leptons and polarized nucleons have found that the spins of quarks and antiquarks account for only about $25 \%$ of the nucleon spin [1]. The gluon helicity distribution and orbital angular momenta are thus essential to the understanding of the nucleon spin. Analyses of the scale dependence of the inclusive nucleon spin structure function [2] and recent semi-inclusive DIS data [3] have coarsely constrained the possible gluon spin contribution. Complementary measurements with strongly interacting probes $[4,5]$ give sensitivity to gluons predominantly via quarkgluon and gluon-gluon scattering contributions [6].

In this Letter we report the first measurement of $A_{\mathrm{LL}}$ for inclusive jet production in polarized proton collisions,

$$
A_{\mathrm{LL}}=\frac{\sigma^{++}-\sigma^{+-}}{\sigma^{++}+\sigma^{+-}},
$$

where $\sigma^{++}$and $\sigma^{+-}$are the inclusive jet cross sections when the two colliding proton beams have equal and opposite helicities, respectively. In addition we report the inclusive jet differential cross section.

In $\mathrm{pQCD}$ the (un-)polarized jet cross section involves a convolution of (un-)polarized quark and gluon distribution functions and the (un-)polarized hard partonic scattering cross section [6,7]. We compare next-to-leading order (NLO) pQCD calculations with the measured cross section to test their applicability and to support their use in constraining the polarized gluon distribution through measurement of $A_{\mathrm{LL}}$. Our data on $A_{\mathrm{LL}}$ are sensitive to gluon polarization for momentum fractions $0.03<x<0.3$.

The data were collected at the Brookhaven Relativistic Heavy Ion Collider (RHIC) with the Solenoidal Tracker at RHIC (STAR) [8] in the years 2003 and 2004 using proton beams of $100 \mathrm{GeV}$ energy. Typical luminosities were $2-5 \times 10^{30} \mathrm{~cm}^{-2} \mathrm{~s}^{-1}$. Spin rotator magnets upstream and downstream of the STAR interaction region (IR) rotated the proton beam spins from and to the stable vertical direction in RHIC to provide collisions with longitudinal polarizations [8]. The helicities alternated for successive bunches of one beam and for successive pairs of bunches of the other beam. Thus STAR recorded collisions with all beam helicity combinations in rapid succession.

The polarization of each beam was measured for each beam fill with RHIC Coulomb-nuclear interference (CNI) proton-carbon polarimeters [9], which were calibrated in situ using a polarized atomic hydrogen gas-jet target [10]. Proton beam polarizations were $30 \%-45 \%$. Nonlongitudinal beam polarization components at the STAR IR were measured continuously with local polarimeters [11] and were no larger than 9\% (absolute).
The STAR detector subsystems [8] of principal interest here are the time projection chamber (TPC), the barrel electromagnetic calorimeter (BEMC), and the beambeam counters (BBC). The TPC tracks charged particles in a $0.5 \mathrm{~T}$ solenoid magnetic field for all azimuthal angles $(\phi)$ and pseudorapidities $|\eta| \lesssim 1.3$. The BEMC is a leadscintillator sampling calorimeter that limited the acceptance in 2003 and 2004, covering all $\phi$ and $0<\eta<1$ with respect to the TPC center. The BBCs are composed of segmented scintillator annuli that span $3.3<|\eta|<5.0$ and measure the proton beam luminosity and transverse polarization components.

Proton collision events were identified by coincident signals from at least one $\mathrm{BBC}$ segment on either side of the IR. The cross section for the BBC coincidence requirement is $26.1 \pm 2.0 \mathrm{mb}$, which is $87 \%$ of the non-singly diffractive pp cross section [12]. The jet data were collected with a highly prescaled minimum bias (MB) trigger, requiring a proton collision event, and a high tower (HT) calorimetric trigger condition. It required, in addition, a signal from at least one BEMC tower of size $\Delta \eta \times \Delta \phi=$ $0.05 \times 0.05$ above a transverse energy $\left(E_{T}\right)$ threshold of $2.2 \mathrm{GeV}$ in $2003(2.2-3.4 \mathrm{GeV}$ at $\eta=0-1$ in 2004). In total $2.1 \times 10^{6} \mathrm{MB}$ and $3.0 \times 10^{6} \mathrm{HT}$ events were analyzed. The integrated luminosity $\int \mathcal{L} d t$ amounts to $0.18(0.12) \mathrm{pb}^{-1}$ for the analyzed 2003 (2004) data.

Jets were reconstructed using a midpoint-cone algorithm [13] that clusters reconstructed TPC tracks and BEMC energy deposits within a cone in $\eta$ and $\phi$ starting from energy seeds of at least $0.5 \mathrm{GeV}$. A cone radius $r_{\text {cone }}=0.4$ was chosen because of the limited BEMC $\eta$ acceptance. Particle tracks with $p_{T}>0.2 \mathrm{GeV} / c$ were considered if they originated from the primary interaction vertex, which was required to be on the beam axis and within $60 \mathrm{~cm}$ from the TPC center to ensure uniform tracking efficiency. Calorimeter towers were considered if their $E_{T}$ exceeded $0.2 \mathrm{GeV}$ after correction for charged hadron contributions determined from TPC tracking. A charged pion (photon) mass was assumed for tracks (towers) in relating energy and momentum. Jets were required to have a reconstructed jet $p_{T}>5 \mathrm{GeV} / c$ and, as a tradeoff between acceptance and effects from acceptance edges, a reconstructed jet axis intersecting the BEMC at nominal $\eta$ between 0.2 and 0.8 . A minimum TPC contribution to the jet energy, $E_{\mathrm{TPC}} / E_{\mathrm{tot}}>0.2(0.1)$ in 2003 (2004), was used to suppress apparent jets from beam background. The jet $p_{T}$ resolution was determined to be $\sim 25 \%$ from the momentum balance of dijet events and from simulation, and motivated the choice of binning. 
Figure 1 compares the measured and simulated jet profile $\Psi\left(\Delta r, r_{\text {cone }}, p_{T}\right)$, defined as the average fraction of jet $E_{T}$ inside a coaxial inner cone of radius $\Delta r<r_{\text {cone }}$, for the MB and HT data separately. The reconstruction software imposed the same trigger requirements as in the data. More than $95 \%$ of the total jet energy is expected to be contained within $r_{\text {cone }}=0.4$. The HT trigger, providing increased selectivity for jets, causes a $p_{T}$ dependent bias toward jets with hard fragments that produce an electromagnetic shower. The $\Psi\left(\Delta r, r_{\text {cone }}, p_{T}\right)$ distributions are well reproduced by PYTHIA-based (v 6.205 [14] "CDF TuneA" settings [15]) Monte Carlo simulations passed through GEANT-based [16] STAR detector simulation. The simulations are used in determining the cross section and to assess effects of the trigger bias on $A_{\mathrm{LL}}$. In the cross section analysis of HT data an $E_{T}$ threshold of $3.5 \mathrm{GeV}$ was imposed on the BEMC trigger tower to ensure a uniform trigger efficiency.

The differential inclusive cross sections were determined separately for the MB and HT data according to

$$
\frac{1}{2 \pi} \frac{d^{2} \sigma}{d \eta d p_{T}}=\frac{1}{2 \pi} \frac{N_{\text {jets }}}{\Delta \eta \Delta p_{T}} \frac{1}{\int \mathcal{L} d t} \frac{1}{c\left(p_{T}\right)},
$$

where $N_{\text {jets }}$ denotes the number of jets observed within a pseudorapidity interval $\Delta \eta$ and a transverse momentum interval $\Delta p_{T}$ at a mean jet $p_{T}$. The correction factors $c\left(p_{T}\right)$ were determined from simulation, and are defined as the ratio of the number of jets reconstructed within a given $p_{T}$ interval in the simulated data to those generated in the PYTHIA final-state particle record. They change monotonically for $\mathrm{HT}$ events from 0.02 at $p_{T}=8.3 \mathrm{GeV} / c$ to 0.79 at $p_{T}=43 \mathrm{GeV} / c$, whereas they are a constant 0.69 for MB events with $p_{T}<12.6 \mathrm{GeV} / c$. Consistent values were obtained with the HERWIG [17] generator. Typically $35 \%-40 \%$ of the jets generated in a given $p_{T}$ interval were reconstructed in the same interval. Reconstructed $p_{T}$ was found to be on average $\sim 20 \%$ larger than generated $p_{T}$ in each reconstructed $p_{T}$ interval, and the difference is taken into account via $c\left(p_{T}\right)$.

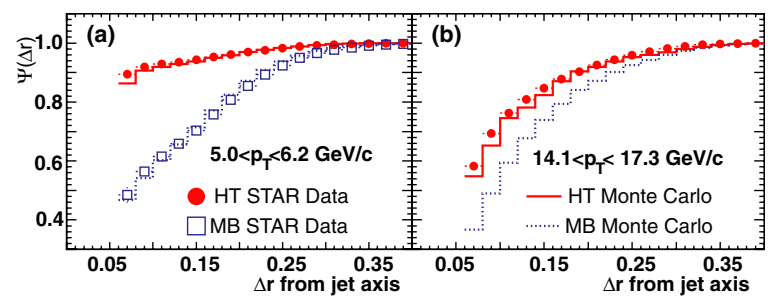

FIG. 1 (color online). Jet profile $\Psi\left(\Delta r, r_{\text {cone }}, p_{T}\right)$ versus inner cone size $\Delta r$ at $r_{\text {cone }}=0.4$ for MB (open squares) and HT (filled circles) data compared with STAR Monte Carlo simulation in two jet $p_{T}$ bins (a) $5.0<p_{T}<6.2$ and (b) $14.1<$ $p_{T}<17.3 \mathrm{GeV} / c$. In (b) the $\mathrm{MB}$ jet yield was too small to measure.
The MB differential cross sections extracted from $1.4 \times$ $10^{3}$ jets collected in 2003 and $1.1 \times 10^{3}$ in 2004 are in good agreement $\left(\chi^{2} / \mathrm{ndf}=0.8\right)$. A $20 \%$ systematic offset for all $p_{T}$ was found between the HT differential cross sections extracted from $43 \times 10^{3}$ and $42 \times 10^{3}$ jets collected in 2003 and 2004. We ascribe this difference to 5\% uncertainty (included in the systematic errors below) in the year-to-year absolute scale of the BEMC calibration, which was changed by a factor of $\sim 2$ between the two years, and to uncertainty in the modeling of temporary BEMC hardware malfunctions. The calibration used $20 \times$ $10^{6} d+$ Au collision events in 2003 and $50 \times 10^{6} \mathrm{Au}+$ $\mathrm{Au}$ events in 2004. The absolute energy scale was set by matching BEMC energy to TPC track momentum for wellcontained showers from $1.5<p<8 \mathrm{GeV} / c$ electrons identified in the TPC. Uncertainties arise in the electron selection, from residual hadronic contamination, and from the limited $d+$ Au statistics.

Figure 2(a) shows the arithmetic average of the 2003 and $2004 \mathrm{MB}$ and HT cross sections versus jet $p_{T}$. The MB and HT data are in good agreement for overlapping jet $p_{T}$ $\left(\chi^{2} / \mathrm{ndf}=1.0\right)$, despite the very different $c\left(p_{T}\right)$. The curve shows the NLO pQCD cross section of Ref. [6] evaluated at equal factorization and renormalization scales, $\mu \equiv \mu_{F}=$ $\mu_{R}=p_{T}$, using the CTEQ6M parton distributions [18]. Figure 2(b) compares data and theory, showing satisfactory agreement over 7 orders of magnitude. The theoretical

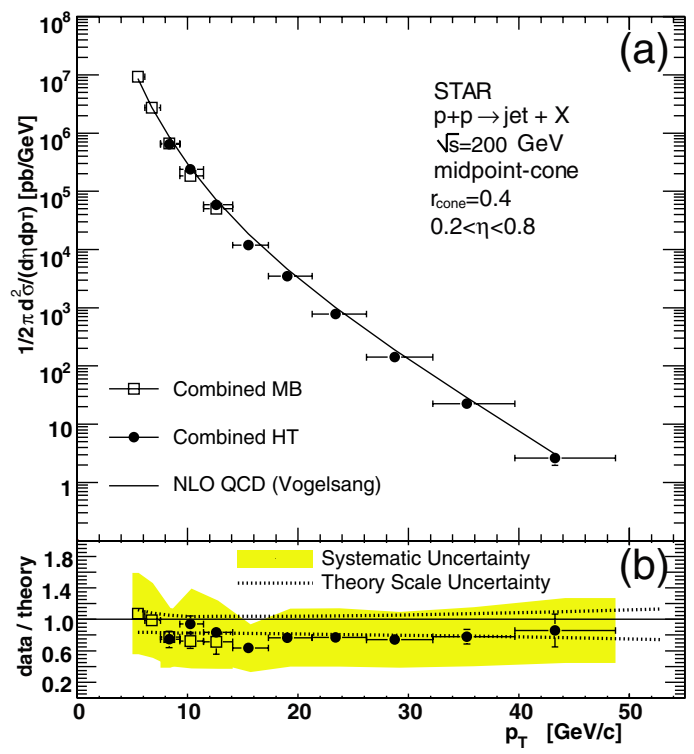

FIG. 2 (color online). (a) Inclusive differential cross section for $p+p \rightarrow$ jet $+X$ at $\sqrt{s}=200 \mathrm{GeV}$ versus jet $p_{T}$ for a jet cone radius of 0.4 . The symbols show MB (open squares) and HT (filled circles) data from the years 2003 and 2004 combined. The horizontal bars indicate the ranges of the $p_{T}$ intervals. The curve shows a NLO calculation [6]. (b) Comparison of theory and data. The band indicates the experimental systematic uncertainty. The upper (lower) dashed line indicates the relative change of the NLO calculation when it is evaluated at $\mu=p_{T} / 2\left(\mu=2 p_{T}\right)$. 
cross section changes by less than $23 \%$ if $\mu$ is varied by a factor of 2 and increases by $1 \%(13 \%)$ at $p_{T}$ of 10 (40) $\mathrm{GeV} / c$ if the CTEQ6.1M distributions are used. The experimental systematic uncertainty amounts to $8 \%$ in the normalization with the BBC and $48 \%$ in the measured yield, consisting of $5 \%$ due to residual beam background, $13 \%$ on $c\left(p_{T}\right)$, and $46 \%$ from a $9 \%$ uncertainty on the jet energy scale. The BEMC calibration and undetected neutral particles dominate in the latter. No corrections were made for the nonperturbative redistribution of energy into and out of the jet by the underlying event and out-of-cone hadronization. We estimate that such corrections would increase the measured differential cross section by $\sim 25 \%$ for $p_{T}>10 \mathrm{GeV} / c$.

The asymmetry $A_{\mathrm{LL}}$ was extracted for $5<p_{T}<$ $17 \mathrm{GeV} / c$ from a HT data sample of about $110 \times 10^{3}$ jets in 2003 and $210 \times 10^{3}$ in 2004 . The sample size is larger than in the cross section analysis, since no BEMC energy threshold was required. The jet yields $N$ were sorted by equal $(++)$ and opposite $(+-)$ beam helicity configurations. The asymmetry was extracted as

$$
A_{\mathrm{LL}}=\frac{\sum\left(P_{1} P_{2}\right)\left(N^{++}-R N^{+-}\right)}{\sum\left(P_{1} P_{2}\right)^{2}\left(N^{++}+R N^{+-}\right)}
$$

where $P_{1,2}$ are the measured proton beam polarizations, $R \simeq 1.1$ is the ratio of measured luminosities for equal and opposite proton beam helicities, and parity violating differences $\lesssim \mathcal{O}\left(10^{-4}\right)$ in the cross sections for different beam helicities are not considered. The sums are performed over runs typically lasting 20 minutes.

The results for $A_{\mathrm{LL}}$ from 2003 and 2004 data are in good agreement $\left(\chi^{2} / \mathrm{ndf}=0.3\right)$. Figure 3 shows the combined $A_{\mathrm{LL}}$ versus jet $p_{T}$, together with the statistical (bars) and systematic (bands) uncertainties.

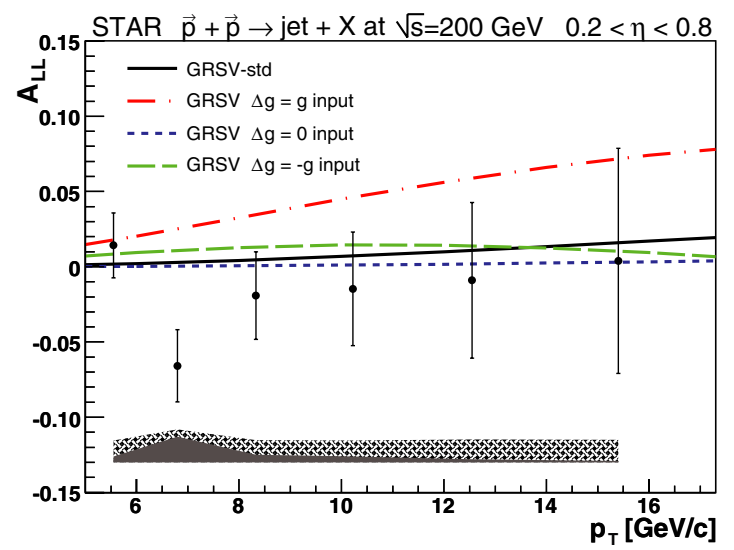

FIG. 3 (color online). The longitudinal double-spin asymmetry $A_{\mathrm{LL}}$ in $\vec{p}+\vec{p} \rightarrow$ jet $+X$ at $\sqrt{s}=200 \mathrm{GeV}$ versus jet $p_{T}$. The uncertainties on the data points are statistical. The gray band indicates the systematic uncertainty from the beam polarization measurement, and the hatched band the total systematic uncertainty. The curves show predictions based on deep-inelastic scattering parametrizations of gluon polarization $[6,18]$.
A $25 \%$ combined scale uncertainty arises from the CNI beam polarization measurement $(22 \%$ in 2003 and an uncorrelated $16 \%$ in 2004) and from the CNI absolute calibration (18\% common to both years).

The uncertainty in $R$ was estimated to be 0.003 using narrow and wide timing requirements for the $\mathrm{BBC}$ coincidence. It takes into account differences in sampling of the longitudinal vertex distribution in the jet analysis and in the relative luminosity measurement, and corresponds to 0.009 uncertainty in $A_{\mathrm{LL}}$. An independent measurement with the zero degree calorimeters (ZDC) [8] gave consistent results to within statistical uncertainties. No double helicity asymmetry of the BBC measurement relative to the ZDC measurement was observed.

Residual nonlongitudinal proton beam polarization at the STAR IR could contaminate the $A_{\mathrm{LL}}$ measurement through an azimuthally uniform two-spin asymmetry [19]. A limit of 0.010 on such contamination was set from local polarimetry data and from two-spin asymmetry measurements with vertically polarized beams.

Beam background occasionally caused BEMC signals not associated with collisions at the IR. Its effect on the jet yields was reduced with the aforementioned selection on $E_{\mathrm{TPC}} / E_{\mathrm{tot}}$. Residual yields were estimated to be no larger than 8\% (5\%) in the 2003 (2004) data from the variation of jet spectra with beam-background conditions monitored with the BBCs when filled and empty beam bunches crossed at the IR. These, combined with asymmetry estimates from beam background dominated samples, resulted in 0.003 uncertainty in $A_{\mathrm{LL}}$.

The bias toward hard fragmentation processes caused by the HT trigger requirement was simulated, as were possible biases introduced by jet reconstruction and jet $p_{T}$ resolution. The resulting $p_{T}$ dependent shifts in $A_{\mathrm{LL}}$ were estimated with the polarized parton distributions of Ref. [20]. Their total is estimated to be less than 0.009 .

Analyses with randomized proton beam helicity configurations and other cross checks including parity violating single-spin asymmetries showed the expected statistical behavior, thus indicating no evidence for beam bunch to bunch or fill to fill systematics in $A_{\mathrm{LL}}$.

The curves in Fig. 3 show theoretical evaluations $[6,18]$ at $\mu=p_{T}$ for the commonly used polarized parton distributions of Ref. [20]. They shift by less than $0.003(0.017)$ at $p_{T}=5.6(15.7) \mathrm{GeV} / c$ if $\mu$ is varied by a factor of 2 . The polarized parton distributions are based on a best fit to polarized inclusive DIS data, the so-called GRSV-standard gluon polarization distribution, and on assumptions of (i) a vanishing gluon polarization $\Delta g\left(x, Q_{0}^{2}\right)=0$, and (ii) maximally positive or negative gluon polarization, $\Delta g\left(x, Q_{0}^{2}\right)=$ $\pm g\left(x, Q_{0}^{2}\right)$, at the initial scale $Q_{0}^{2}=0.4 \mathrm{GeV}^{2} / c^{2}$ in the analysis [20]. Alternative parametrizations [21] are within the range spanned by (ii). Our data fall below the $\Delta g\left(x, Q_{0}^{2}\right)=g\left(x, Q_{0}^{2}\right)$ evaluation $\left(\chi^{2} /\right.$ ndf $\left.\simeq 3\right)$ and are consistent with the other evaluations, in qualitative agree- 
ment with Refs. [3-5]. The results thus disfavor large and positive gluon polarization, proposed [22] originally to explain the small quark spin contribution to the proton spin.

In summary, we report the first measurement of the longitudinal double-spin asymmetry $A_{\mathrm{LL}}$ for inclusive jets with transverse jet momenta of $5<p_{T}<17 \mathrm{GeV} / c$ produced at midrapidity in polarized proton collisions at $\sqrt{s}=200 \mathrm{GeV}$. The jet cross section was determined for $5<p_{T}<50 \mathrm{GeV} / c$ and is described by NLO pQCD evaluations over 7 orders of magnitude. The asymmetries $A_{\mathrm{LL}}$ are consistent with NLO pQCD calculations utilizing polarized quark and gluon distributions from inclusive DIS analyses, and disfavor at $98 \%$ C.L. large positive values of gluon polarization in the polarized nucleon.

We thank the RHIC Operations Group and RCF at BNL, and the NERSC Center at LBNL for their support. This work was supported in part by the Offices of NP and HEP within the U S. DOE Office of Science; the U. S. NSF; the BMBF of Germany; CNRS/IN2P3, RA, RPL, and EMN of France; EPSRC of the United Kingdom; FAPESP of Brazil; the Russian Ministry of Science and Technology; the Ministry of Education and the NNSFC of China; IRP and GA of the Czech Republic, FOM of the Netherlands, DAE, DST, and CSIR of the Government of India; Swiss NSF; the Polish State Committee for Scientific Research; SRDA of Slovakia, and the Korea Sci. \& Eng. Foundation.

[1] J. Ashman et al. (EMC Collaboration), Nucl. Phys. B328, 1 (1989); B. W. Filippone and X. Ji, Adv. Nucl. Phys. 26, 1 (2001), and references therein.

[2] B. Adeva et al. (SMC Collaboration), Phys. Rev. D 58, 112002
(1998); P. L. Anthony et al. (E155 Collaboration), Phys. Lett. B 493, 19 (2000).

[3] A. Airapetian et al. (HERMES Collaboration), Phys. Rev. Lett. 84, 2584 (2000); B. Adeva et al. (SMC Collaboration), Phys. Rev. D 70, 012002 (2004); E. S. Ageev et al. (COMPASS Collaboration), Phys. Lett. B 633, 25 (2006).

[4] D. L. Adams et al. (E581/E704 Collaboration), Phys. Lett. B 261, 197 (1991); Phys. Lett. B 336, 269 (1994).

[5] S.S. Adler et al. (PHENIX Collaboration), Phys. Rev. Lett. 93, 202002 (2004); Phys. Rev. D 73, 091102(R) (2006).

[6] B. Jäger et al., Phys. Rev. D 70, 034010 (2004).

[7] G. Bunce et al., Annu. Rev. Nucl. Part. Sci. 50, 525 (2000).

[8] Special Issue on RHIC and Its Detectors, edited by M. Harrison, T. Ludlam, and S. Ozaki [Nucl. Instrum. Methods Phys. Res., Sect. A 499, Nos. 2-3 (2003)].

[9] O. Jinnouchi et al., RHIC/CAD Accelerator Physics Note 171 (2004).

[10] H. Okada et al., hep-ex/0601001 [Phys. Lett. B (to be published)].

[11] J. Kiryluk et al., hep-ex/0501072.

[12] J. Adams et al. (STAR Collaboration), Phys. Rev. Lett. 91, 172302 (2003).

[13] G. Blazey et al., hep-ex/0005012.

[14] T. Sjostrand et al., Comput. Phys. Commun. 135, 238 (2001).

[15] R. D. Field et al., hep-ph/0510198.

[16] GEANT 3.21, CERN program library.

[17] G. Corcella et al., J. High Energy Phys. 01 (2001) 010.

[18] J. Pumplin et al., J. High Energy Phys. 07 (2002) 012.

[19] H. O. Meyer et al., Phys. Rev. Lett. 81, 3096 (1998).

[20] M. Glück et al., Phys. Rev. D 63, 094005 (2001).

[21] See, e.g., M. Hirai et al., Phys. Rev. D 69, 054021 (2004), and references therein.

[22] G. Altarelli and G. G. Ross, Phys. Lett. B 212, 391 (1988); R. D. Karlitz et al., Phys. Lett. B 214, 229 (1988). 